\title{
Method S2
}

We provide in this supplementary the basic definition of the notion of false-positive rate, or type-Ierror rate, and power. Considering a test looking at a null hypothesis $H_{0}$ and an alternative hypothesis $H_{1}$ under the significance level $\alpha$ we can draw the following table describing the possible outcomes.

\begin{tabular}{|l|c|c|}
\hline & $H_{0}$ is accepted & $H_{0}$ is rejected \\
\hline$H_{0}$ is true & True Negative $(1-\alpha)$ & False Positive / Type-I error rate $(\alpha)$ \\
\hline$H_{0}$ is false & False Negative / Type-II error rate $(\beta)$ & True Positive / Power $(1-\beta)$ \\
\hline
\end{tabular}

Definition: The false positive rate is the probability of rejecting the null hypothesis when this hypothesis is true, i.e. the probability of detecting a SNP associated with the disease when it is not the case. It corresponds to $\mathbb{P}_{H_{0}}$ (rejecting $\left.H_{0}\right)$. All the tests are conducted at the same level $\alpha$ which means that we expect a false positive rate of $\alpha$ as well.

Definition: The statistical power is defined as the probability of rejecting the null hypothesis when the alternative hypothesis is true, i.e. the probability of detecting an association when there is actually

one. Formally speaking, the statistical power corresponds to $\mathbb{P}_{H_{1}}$ (rejecting $H_{0}$ ) and is function of the test significance level $\alpha$. 